\title{
MHD natural convection nanofluid flows in a wavy trapezoidal porous enclosure with differentially heated side walls
}

\author{
E Suresh Reddy* and Satyananda Panda \\ Department of Mathematics, National Institute of Technology, Calicut, Kerala, India.
}

Submitted: 11 September 2018; Revised: 26 April 2019; Accepted: 23 August 2019

\begin{abstract}
Flow analysis of the $\mathrm{Al}_{2} \mathrm{O}_{3}$ - water nanofluid in a porous trapezoidal enclosure is deliberated in this research work. The enclosure is bounded by four differentially heated walls with the bottom wall considered as wavy. The viscous and Joule dissipation effects are taken into account. The governing equations of the flow are non-dimensionalised, and the Galerkin finite element method is employed to solve the resulting unsteady initial and boundary value problem using Taylor-Hood elements to avoid instabilities. The numerical scheme is validated with available literature for a simplified case of a problem, and the results are found to be in good agreement. The influence of Darcy number and nanoparticle volume fraction on the dimensionless streamlines and isotherms are plotted and illustrated in detail. The rate of heat transfers near the bottom wall in a porous medium is analysed through simulated data. It is found that the heat transfer rate increases in the presence of a porous domain. Further, a sensitivity analysis has been performed using Response Surface Methodology (RSM) to identify the pertinent parameters of the problem such as Hartmann number, Darcy number, and nanoparticles whose variation have an important influence on the average Nusselt number. The analysis reveals that the Darcy number, Hartmann number and the nanoparticle volume fraction are related to the average Nusselt number. Thus, the finding suggests that the porous medium has a significant role in enhancing the heat transfer in a flow of MHD nanofluid within a wavy trapezoidal enclosure.
\end{abstract}

Keywords: Finite element method, MHD nanofluid flow, natural convection, porous medium, trapezoidal wavy enclosure, viscous and Joule dissipation.

\section{INTRODUCTION}

The extensive applications of natural convection nanofluid flow inside an enclosure in various fields of science and engineering have attracted the attention of numerous researchers. Particular models of such flows happen in the design of solar collectors, electronic cooling processes, lubrication technologies, food processing, and nuclear reactors. In this process, the combination of a base fluid like water, oil, kerosene, and the nanoparticle such as $\mathrm{Al}, \mathrm{Cu}, \mathrm{Ag}$ alters the thermal properties of the liquid and hence enhances the performance of the cooling system (Choi \& Eastman, 1995; Daungthongsuk \& Wongwises, 2009). The nanofluid in an enclosure with differentially heated side walls induces an inhomogeneous temperature field in the liquid resulting in convection effects that cause the motion of the fluid. A considerable amount of literature pertaining to this phenomena can be found in regular enclosures, such as trapezoidal enclosure (Qu et al., 2000; Suh et al., 2001; Roslan et al., 2011; Mahmoudi et al., 2013), square enclosure (Ternik et al., 2012; Ternik \& Rudilf, 2014), and rectangular enclosure (Hwang et al., 2007; Salari et al., 2017).

Thus, many of the research mentioned as earlier work are devoted to the study of heat transfer of nanofluid flow in an enclosure with flat walls. It is understood that an enclosure with a wavy wall improves heat transfer when

\footnotetext{
*Corresponding author (sreddy4429@gmail.com; (iD https://orcid.org/0000-0002-5924-5235)
} 
compared to a flat wall. Cho et al. (2012) have numerically examined the flow and heat transfer in a wavy wall enclosure in the presence of copper nanoparticles. It is shown that the mean Nusselt number increases with the increase of solid volume fraction of nanoparticles. The study further found that the suitable geometry conditions corresponding to the Rayleigh number can optimise the mean Nusselt number. Singh and Bhargava (2014) have employed the meshfree Galerkin numerical method to analyse the effects of geometry of the heat transfer surface, the Prandtl number and the Rayleigh number on the heat transfer analysis of a single phase fluid in a wavy enclosure.

It is observed that the rate of heat transfer is higher for wavy surfaces as compared to smooth surfaces. Recently, Job and Gunakala (2016) used the finite element method to analyse the unsteady buoyancy-driven nanofluid flows inside an enclosure, which is trapezoid with the wavy bottom wall. It was observed that the rise in amplitude of the wavy wall results in a reduction of the strength of the flow circulation and increment in temperature. The authors have also considered the magnetic effect into the problem, which has many practical applications associated with the nanofluid flow and heat transfer. It was observed that the temperature of the aluminawater based nanofluid increases with the rise in the electromagnetic force. More recently, the problem of natural convection heat transfer and fluid flow of $\mathrm{Al}_{2} \mathrm{O}_{3}$ water-based nanofluid inside a wavy enclosure was investigated using ANSYS-FLUENT by Leonard et al. (2017). The investigation revealed that increment in surface waviness, solid volume fraction and aspect ratio helps to enhance the heat transfer.

Flow in porous media has been a subject of active research for the last four to five decades. Study of fluid and heat flow within porous media is also of vital importance in many other fields of science and engineering, such as drying of biological materials and biomedical studies. However, some questions regarding the flow of nanofluid inside a porous wavy enclosure with differentially heated walls remain unanswered. For example, how porous matrix affects the flow and heat transfer of nanofluid in a wavy enclosure? This study attempts to address these questions by solving the nanofluid system in a porous wavy enclosure with differentially heated boundary walls. Alhashash et al. (2017) have investigated the effects of nanoparticle concentration on unsteady natural convection filled with a fluid-saturated porous domain although the discussion was limited to a square porous domain within the framework of Boussinesq approximation. Sensitivity analysis applies in engineering problems to determine how different values of an independent variable affect the desired output. This technique is advantageous when attempting to assess the impact of several influence parameters on outputs of a problem. Some researchers used this analysis in different thermal engineering problems. Rashidi et al. (2015a) used the sensitivity analysis for porous solar heat exchangers by response surface methodology. In another research, Rashidi et al. (2015b; 2016) performed the optimisation and sensitivity analyses for convective heat transfer of water-alumina nanofluid flow over an equilateral triangular cylinder with different orientations. They selected the substantial volume fraction of nanoparticles, Reynolds number, and the orientation of the obstacle as input parameters. Further, the drag coefficient and Nusselt number were used as output variables. They reported that the Nusselt number and drag coefficient are more sensitive to the orientation of the obstacle in comparison to the Reynolds number and solid volume fractions of nanoparticles.

The present analysis considers a two-dimensional system of nanofluid flow in a wavy porous enclosure governed by the Darcy model. This model extends the analysis of Job and Gunakala (2016) by incorporating porous effects which has a significant influence on heat transfer analysis on the flow of nanofluid in a trapezoidal enclosure. The effect of viscous and Joule dissipation are also included for the thermal analysis. The two-dimensional model equations are the system of coupled partial differential equations on a complex domain, and an efficient numerical scheme is needed for the numerical solution of the problem. In this study, we have used the Galerkin finite element method with Taylor-Hood elements (Donea \& Huerta, 2003; Reddy, 2004) to guarantee the stability of the numerical scheme. A discussion is provided for several parameters governing the problem. The dimensionless profiles of stream function the temperature for different parameters have been discussed. Additionally, a useful parameter analysis is performed by using the Response Surface Methodology (RSM) to identify the sensitive parameters which influence the average Nusselt number.

We consider the flow of time-dependent twodimensional nanofluid through a porous medium in a bounded trapezoidal enclosure $\Omega \subset R^{2}$. The domain $\Omega$ is bounded by four rigid boundary walls, $\partial \Omega=\Gamma_{1} \cup \Gamma_{2} \cup \Gamma_{3} \cup \Gamma_{4}$ with $\Gamma_{i} \cap \Gamma_{j}=\emptyset$ for $i \neq j$ with $i, j \in\{1,2,3,4\}$

The distance of the top wall the enclosure height is $h$, and the top wall is joining with left and right walls at an angle of $45^{\circ}$. The schematic of the flow configuration is given in Figure 1. 


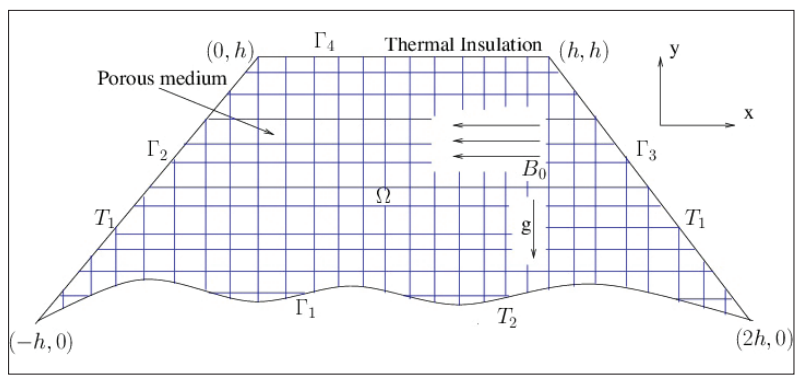

Figure 1: Schematic diagram of the domain

The bottom wall of the enclosure (Figure 1) is considered as a wavy curve with domain $[-h, 2 h]$, amplitude $a h(a \in[0,0.1])$ with period $h$, and given by,

$f(x)=a h\left\{1+\cos \left[\frac{2 \pi(x+h)}{h}+\pi\right]\right\}, \quad-h \leq x \leq 2 h$

Initially, the fluid in the enclosure is kept at a constant temperature $T_{1}$. With the advancement of time, i.e, $t>0$, the bottom curve wall started to get heated and attain the temperature $T_{2}$ which is greater than $T_{1}$. The left and right walls are maintained at a temperature $T_{1}$. The top wall is considered insulated thermally so that heat transfer cannot take place through the wall as shown in Figure 1. Due to uneven distribution of temperature inside the domain walls, a temperature gradient develops which induces flow due to the convection effects. A magnetic field of strength $B_{0}$ is applied in the negative $x$-direction that causes Joule dissipation effect. The viscous dissipation effect is included in the problem due to the temperature difference.

The following assumptions are made in the analysis of the underlying problem:

- The flow is considered to be two-dimensional and incompressible

- The volume charge density is minimal compared to the Lorentz force

- The effects of advection of magnetic field are relatively negligible

- The effects of thermal radiation are negligible

- The Boussinesq approximation is applicable to approximate the density

- The nanoparticles concentration is small, and they have a uniform size and spherical shape

The governing equations for the mass and momentum of the nanofluid governed by the Darcy model with underlying assumptions are:

$$
\begin{aligned}
\frac{\partial u}{\partial x}+\frac{\partial v}{\partial y}=0 & \\
\rho_{n f}\left(\frac{\partial u}{\partial t}+u \frac{\partial u}{\partial x}+v \frac{\partial u}{\partial y}\right) & =-\frac{\partial p}{\partial x}+\mu_{n f}\left(\frac{\partial^{2} u}{\partial x^{2}}+\frac{\partial^{2} u}{\partial y^{2}}\right) \\
& -\frac{\mu_{n f}}{K} u \\
\rho_{n f}\left(\frac{\partial v}{\partial t}+u \frac{\partial v}{\partial x}+v \frac{\partial v}{\partial y}\right)= & -\frac{\partial p}{\partial y}+\mu_{n f}\left(\frac{\partial^{2} v}{\partial x^{2}}+\frac{\partial^{2} v}{\partial y^{2}}\right)
\end{aligned}
$$

$$
-\frac{\mu_{n f}}{K} v-\sigma_{n f} B_{0}^{2} v+(\rho \beta)_{n f} g\left(T-T_{1}\right)
$$

and following Al-Hadhrami et al. (2003), the temperature equation can be written as:

$$
\begin{aligned}
\left(\rho c_{p}\right)_{n f}\left(\frac{\partial T}{\partial t}+u \frac{\partial T}{\partial x}\right. & \left.+v \frac{\partial T}{\partial y}\right)=k_{n f}\left(\frac{\partial^{2} T}{\partial x^{2}}+\frac{\partial^{2} T}{\partial y^{2}}\right) \\
& +\sigma_{n f} B_{0}^{2} v^{2}+\frac{\mu_{n f}}{K}\left(u^{2}+v^{2}\right) \\
& +\mu_{n f}\left(2\left(\frac{\partial u}{\partial x}\right)^{2}+2\left(\frac{\partial v}{\partial y}\right)^{2}+\left(\frac{\partial v}{\partial x}+\frac{\partial u}{\partial y}\right)^{2}\right)
\end{aligned}
$$

with initial condition

$u=0, v=0, T=T_{1}$ on $\Omega \times\{0\}$,

and boundary conditions

$u=0, v=0, T=T_{2}$ on $\Gamma_{1} \times\left(0, t_{1}\right]$,

$u=0, v=0, \frac{\partial T}{\partial y}=0$ on $\Gamma_{4} \times\left(0, t_{1}\right]$

$u=0, v=0, T=T_{1}$ on $\left(\Gamma_{2} \cup \Gamma_{3}\right) \times\left(0, t_{1}\right]$,

where $t_{1}$ is the length of the time interval.

The initial condition, equation (6) is due to the fluid at rest with an initial temperature $T_{1}$, and the boundary conditions equations (7)-(9) are arising due to noslip boundary conditions applied on left, top, bottom, and right walls. The temperature boundary effects are modelled by prescribing fixed temperature $T_{1}$ at the left and right walls, and the curved bottom wall is maintained at temperature $T_{2}$. The top wall is assumed to be insulated.

It may be recorded that for the non-porous medium, i.e., $K \rightarrow \infty$, model equations (2)-(5) together with initial and boundary conditions equations (6)-(9) are the same as those obtained by Job and Gunakala (2016). 
Following Minkowycz et al. (2013), thermophysical properties of nanofluids that are presented in governing equations are given as follows:

Density: $\quad \rho_{n f}=(1-\phi) \rho_{f}+\phi \rho_{s}$

Heat Capacity: $\quad\left(\rho c_{p}\right)_{n f}=(1-\phi)\left(\rho c_{p}\right)_{f}+\phi\left(\rho c_{p}\right)_{s}$

Thermal Expansion Coefficient:

$$
(\rho \beta)_{n f}=(1-\phi)(\rho \beta)_{f}+\phi(\rho \beta)_{s}
$$

Viscosity: $\quad \mu_{n f}=\frac{\mu_{f}}{(1-\phi)^{2.5}}$

Electrical Conductivity:

$$
\sigma_{n f}=\sigma_{f}\left(1+\frac{3\left(\sigma_{s} / \sigma_{f}-1\right) \phi}{\left(\sigma_{s} / \sigma_{f}+2\right)-\left(\sigma_{s} / \sigma_{f}-1\right) \phi}\right)
$$

Thermal Conductivity: $k_{n f}=k_{f} \frac{k_{s}+2 k_{f}+2 \phi\left(k_{s}-k_{f}\right)}{k_{s}+2 k_{f}-\phi\left(k_{s}-k_{f}\right)}$

It is convenient at this stage to introduce the following dimensionless variables (in corresponding hat):

$(\hat{x}, \hat{y})=\frac{(x, y)}{h},(\hat{u}, \hat{v})=\frac{\left(\rho c_{p}\right)_{f} h}{k_{f}}(u, v), \hat{t}=\frac{k_{f}}{\left(\rho c_{p}\right)_{f} h^{2}} t$,

and $\hat{p}=\frac{\left(\rho c_{p}\right)_{f}^{2} h^{2}}{\rho_{f} k_{f}^{2}} p, \quad \hat{T}=\frac{T-T_{1}}{T_{2}-T_{1}}$

The resulting non-dimensionalised equations obtained from equations (2)-(9) using scaling relation equation (10), and by dropping the hat sign are:

$$
\frac{\partial u}{\partial x}+\frac{\partial v}{\partial y}=0
$$

$\frac{\partial u}{\partial t}+u \frac{\partial u}{\partial x}+v \frac{\partial u}{\partial y}=-\frac{\rho_{f}}{\rho_{n f}} \frac{\partial p}{\partial x}+\frac{\mu_{n f}}{\mu_{f}} \frac{\rho_{f}}{\rho_{n f}} \operatorname{Pr}\left(\frac{\partial^{2} u}{\partial x^{2}}+\frac{\partial^{2} u}{\partial y^{2}}\right)$

$$
-\frac{\mu_{n f}}{\mu_{f}} \frac{\rho_{f}}{\rho_{n f}} \frac{P r}{D a} u
$$

$$
\begin{aligned}
\frac{\partial v}{\partial t}+u \frac{\partial v}{\partial x}+v \frac{\partial v}{\partial y} & =-\frac{\rho_{f}}{\rho_{n f}} \frac{\partial p}{\partial y}+\frac{\mu_{n f}}{\mu_{f}} \frac{\rho_{f}}{\rho_{n f}} \operatorname{Pr}\left(\frac{\partial^{2} v}{\partial x^{2}}+\frac{\partial^{2} v}{\partial y^{2}}\right) \\
& -\frac{\mu_{n f}}{\mu_{f}} \frac{\rho_{f}}{\rho_{n f}} \frac{\operatorname{Pr}}{D a} v-\operatorname{Ha}^{2} \operatorname{Pr} \frac{\sigma_{n f}}{\sigma_{f}} \frac{\rho_{f}}{\rho_{n f}} v \\
& +\operatorname{RaPr} \frac{(\rho \beta)_{n f}}{(\rho \beta)_{f}} \frac{\rho_{f}}{\rho_{n f}} T
\end{aligned}
$$

$$
\begin{aligned}
\frac{\partial T}{\partial t}+u \frac{\partial T}{\partial x}+v \frac{\partial T}{\partial y} & =\frac{k_{n f}}{k_{f}} \frac{\left(\rho c_{p}\right)_{f}}{\left(\rho c_{p}\right)_{n f}}\left(\frac{\partial^{2} T}{\partial x^{2}}+\frac{\partial^{2} T}{\partial y^{2}}\right) \\
& +H a^{2} E c \frac{\left(\rho c_{p}\right)_{f}}{\left(\rho c_{p}\right)_{n f}} \frac{\sigma_{n f}}{\sigma_{f}} v^{2} \\
& +\frac{\mu_{n f}}{\mu_{f}} \frac{\left(\rho c_{p}\right)_{f}}{\left(\rho c_{p}\right)_{n f}} E c\left(2\left(\frac{\partial u}{\partial x}\right)^{2}+2\left(\frac{\partial v}{\partial y}\right)^{2}\right. \\
& \left.+\left(\frac{\partial v}{\partial x}+\frac{\partial u}{\partial y}\right)^{2}+\frac{1}{D a}\left(u^{2}+v^{2}\right)\right) .
\end{aligned}
$$

with initial condition

$u=v=0, T=0$ on $\Omega \times\{0\}$,

and boundary conditions

$$
\begin{aligned}
& u=0, v=0, T=1 \text { on } \Gamma_{1} \times\left(0, t_{1}\right], \\
& u=0, v=0, \frac{\partial T}{\partial y}=0 \text { on } \Gamma_{4} \times\left(0, t_{1}\right], \\
& u=0, v=0, T=0 \text { on }\left(\Gamma_{2} \cup \Gamma_{3}\right) \times\left(0, t_{1}\right],
\end{aligned}
$$

where $t_{1}$ is the dimensionless length of the time interval.

The Rayleigh number $\mathrm{Ra}$ is defined in terms of Prandtl number, i.e., $\mathrm{Ra}=(\rho \beta)_{f} \rho_{f} g\left(T_{2}-T_{1}\right) h^{3} \operatorname{Pr} / \mu_{f}^{2}$, where $\operatorname{Pr}=\mu_{f}\left(\rho c_{p}\right)_{f} / \rho_{f} k_{f}$ is the Prandtl number. The non-dimensional Hartmann number $\mathrm{Ha}$ that characterises the magnetic and viscous forces is defined by $\mathrm{Ha}^{2}=\sigma_{f} B_{0}^{2} h^{2} / \mu_{f}$. The dimensionless number $\mathrm{Ec}=\mu_{f} k_{f} /\left(\rho c_{p}\right)_{f}^{2}\left(T_{2}-T_{1}\right) h^{2}$ is the Eckert number, and $\mathrm{Da}=K / h^{2}$ is the Darcy number.

The fluid pattern can be visualised in terms of stream function $\psi$ obtained from the horizontal and vertical velocities, and their relationships are:

$u=\frac{\partial \psi}{\partial y} ; \quad v=-\frac{\partial \psi}{\partial x}$

Simplification of equation (19) implies the governing equation for stream function as

$\frac{\partial^{2} \psi}{\partial x^{2}}+\frac{\partial^{2} \psi}{\partial y^{2}}=\frac{\partial u}{\partial y}-\frac{\partial v}{\partial x}$ 
Since the flow does not cross the fixed walls, the noslip boundary condition is used for all boundaries, i.e., $\psi=0$ on $\partial \Omega \times\left(0, t_{1}\right]$.

In the proposed thermal system, we investigate the average Nusselt number to determine the enhancement of heat transfer due to convection over conduction alone, i.e., the ratio of convection heat transfer and conductive heat transfer normal to the wavy bottom wall $\left(\Gamma_{1}\right)$. The average dimensionless Nusselt number is defined as

$N u_{a v}=-\frac{\int_{\Gamma_{1}}\left(\frac{\partial T}{\partial n}\right)_{\Gamma_{1}} d l}{\int_{\Gamma_{1}}(1) d l}$,

where $l$ is the length of the bottom wall.

\section{METHODOLOGY}

\section{Finite element method}

The finite element method is used in this paper for calculating the primitive variables $u, v, p, \psi$, and $T$. The Galerkin method stands on the variational formulation of the problem in a suitable function space and determines discrete approximations in certain finite dimensional subspaces consisting of piecewise polynomial functions. In the context of the finite element method, there are a few possible combinations of the velocity-pressure pair that satisfies the LBB (Ladyzhenskaya-BabuskaBrezzi) compatibility condition and leads to the result. The triangular Taylor-Hood element (Taylor \& Hood, 1973) happens to be the most regular one in which they only make use of the base nodes. The interpolations of velocity and pressure are continuous at each element but are piecewise continuous over the entire domain. TaylorHood elements can achieve quadratic convergence.

The Taylor-Hood element is significant in approximating pressure by linear polynomials and other unknowns by quadratic polynomials. The weighted residual method helps us to minimise the residual by converting the non-dimensional equations (11)-(14) and equation (20) into a weak formulation. In this scheme, according to Taylor-Hood element, velocity, temperature, and stream function are to be approximated by the second order polynomial and pressure with linear polynomial at each triangular element, i.e., the solution is approximated at each local element as,

$$
\begin{aligned}
& u=\sum_{i=1}^{6} u_{i} N_{i}, \quad v=\sum_{i=1}^{6} v_{i} N_{i}, \quad T=\sum_{i=1}^{6} T_{i} N_{i}, \\
& \psi=\sum_{i=1}^{6} \psi_{i} N_{i}, \quad P=\sum_{i=1}^{3} P_{i} S_{i}
\end{aligned}
$$

with

$$
\begin{aligned}
& N_{1}=L_{1}\left(2 L_{1}-1\right), \quad N_{2}=L_{2}\left(2 L_{2}-1\right), \quad N_{3}=L_{3}\left(2 L_{3}-1\right) \\
& N_{4}=4 L_{1} L_{2}, \quad N_{5}=4 L_{2} L_{3}, \quad N_{6}=4 L_{1} L_{3} \\
& S_{1}=L_{1}, \quad S_{2}=L_{2}, \quad S_{3}=L_{3}=1-L_{1}-L_{2},
\end{aligned}
$$

where $L_{1}, L_{2}, L_{3}$ are all area coordinates of the triangle. Let us divide the whole domain $\Omega$ into a family $\tau_{h}$ of regular finite triangular elements $\Omega_{e}$. In Galerkin scheme we chose weight function $S$ and $N$ as $N=N_{i}, i=1, \ldots, 6$, $S=S_{j}, j=1,2,3$. The equations obtained by weak formulation are

$$
\left\langle\frac{\partial u}{\partial x}+\frac{\partial v}{\partial y}, S\right\rangle=0
$$

$$
\begin{gathered}
\left\langle\frac{\partial u}{\partial t}+u \frac{\partial u}{\partial x}+v \frac{\partial u}{\partial y}, N\right\rangle-\frac{\rho_{f}}{\rho_{n f}}\left\langle p, \frac{\partial N}{\partial x}\right\rangle+\frac{\mu_{n f}}{\mu_{f}} \frac{\rho_{f}}{\rho_{n f}} \operatorname{Pr}\left(\left\langle\frac{\partial u}{\partial x}, \frac{\partial N}{\partial x}\right\rangle\right. \\
\left.+\left\langle\frac{\partial u}{\partial y}, \frac{\partial N}{\partial y}\right\rangle\right)+\frac{\mu_{n f}}{\mu_{f}} \frac{\rho_{f}}{\rho_{n f}} \frac{\operatorname{Pr}}{D a}\langle u, N\rangle=0 \\
\left\langle\frac{\partial v}{\partial t}+u \frac{\partial v}{\partial x}+v \frac{\partial v}{\partial y}, N\right\rangle-\frac{\rho_{f}}{\rho_{n f}}\left\langle p, \frac{\partial N}{\partial y}\right\rangle \\
+\frac{\mu_{n f}}{\mu_{f}} \frac{\rho_{f}}{\rho_{n f}} \operatorname{Pr}\left(\left\langle\frac{\partial v}{\partial x}, \frac{\partial N}{\partial x}\right\rangle+\left\langle\frac{\partial v}{\partial y}, \frac{\partial N}{\partial y}\right\rangle\right) \\
-\operatorname{RaPr} \frac{(\rho \beta)_{n f}}{(\rho \beta)_{f}} \frac{\rho_{f}}{\rho_{n f}}\langle T, N\rangle+\frac{\mu_{n f}}{\mu_{f}} \frac{\rho_{f}}{\rho_{n f}} \frac{\operatorname{Pr}}{D a}\langle v, N\rangle \\
+\operatorname{Ha} a^{2} \operatorname{Pr} \frac{\sigma_{n f}}{\sigma_{f}} \frac{\rho_{f}}{\rho_{n f}}\langle v, N\rangle=0
\end{gathered}
$$

$$
\begin{aligned}
& \left\langle\frac{\partial T}{\partial t}+u \frac{\partial T}{\partial x}+v \frac{\partial T}{\partial y}, N\right\rangle+\frac{k_{n f}}{k_{f}} \frac{\left(\rho c_{p}\right)_{f}}{\left(\rho c_{p}\right)_{n f}}\left(\left\langle\frac{\partial T}{\partial x}, \frac{\partial N}{\partial x}\right\rangle+\left\langle\frac{\partial T}{\partial y}, \frac{\partial N}{\partial y}\right\rangle\right) \\
& -H a^{2} E c \frac{\left(\rho c_{p}\right)_{f}}{\left(\rho c_{p}\right)_{n f}} \frac{\sigma_{n f}}{\sigma_{f}}\left\langle v^{2}, N\right\rangle-\frac{\mu_{n f}}{\mu_{f}} \frac{\left(\rho c_{p}\right)_{f}}{\left(\rho c_{p}\right)_{n f}} \frac{E c}{D a}\left\langle u^{2}+v^{2}, N\right\rangle \\
& -\frac{\mu_{n f}}{\mu_{f}} \frac{\left(\rho c_{p}\right)_{f}}{\left(\rho c_{p}\right)_{n f}} E c\left\langle 2\left(\frac{\partial u}{\partial x}\right)^{2}+2\left(\frac{\partial u}{\partial y}\right)^{2}+\left(\frac{\partial v}{\partial x}+\frac{\partial u}{\partial y}\right)^{2}, N\right\rangle=0
\end{aligned}
$$

$$
\left\langle\frac{\partial \psi}{\partial x}, \frac{\partial N}{\partial x}\right\rangle+\left\langle\frac{\partial \psi}{\partial y}, \frac{\partial N}{\partial y}\right\rangle+\left\langle\frac{\partial u}{\partial y}-\frac{\partial v}{\partial x}, N\right\rangle=0
$$

The discrete equations from equations (21)-(25) are the first order ordinary differential equations with respect to time. The Crank-Nicolson scheme, which has secondorder accuracy is applied to discretise the equations in time. The resulting nonlinear system of algebraic 
equations along with initial and boundary conditions from equations (15)-(18) are solved using the Newton Raphson iterative scheme, which converges with a maximum error less than $10^{-6}$.

In the present study, we consider water as the base fluid, and $\mathrm{Al}_{2} \mathrm{O}_{3}$ nanoparticles are dispersed into it. The nanofluid thermophysical properties are approximated with the help of thermophysical properties of water and $\mathrm{Al}_{2} \mathrm{O}_{3}$ at $25^{\circ} \mathrm{C}$ (Sadoughi et al., 1973) which are given in Table 1.

Table 1: Thermophysical properties of pure water, $\mathrm{Al}_{2} \mathrm{O}_{3}$ at $\mathrm{T}=25^{\circ}$

\begin{tabular}{lcc}
\hline Parameter & Pure water & $\mathrm{Al}_{2} \mathrm{O}_{3}$ \\
\hline$\rho\left(\mathrm{kgm}^{-3}\right)$ & 997.1 & 3970 \\
$C_{p}\left(\mathrm{Jkg}^{-1} \mathrm{~K}^{-1}\right)$ & 4179 & 765 \\
$k\left(\mathrm{Wm}^{-1} \mathrm{~K}^{-1}\right)$ & 0.613 & 40 \\
$\beta\left(\mathrm{K}^{-1}\right)$ & $21 \times 10^{-5}$ & 0.85 \\
$\sigma\left(\Omega^{-1} \mathrm{~m}^{-1}\right)$ & 0.05 & $1 \times 10^{-10}$ \\
\hline
\end{tabular}

Mesh generation of the given domain is done by a software Gmsh. A generation consists of 3775 elements. The degrees of freedom for velocity nodes and pressure nodes per each element is six and three, respectively. By fixing the non-dimensional Prandtl number $P r=6.2$, Eckert number $E c=10^{-5}$, time $t_{d}=1$, Rayleigh number $R a=100$ and Hartmann number $H a=100$, the effect of the porous medium, solid volume fraction and time inside the enclosure with an amplitude of $a=0.1$ is studied. The resulting discretised equations are implemented in Matlab.

To demonstrate successful implementation of the proposed finite element algorithm, the numerical results obtained for an average Nusselt number with $(\mathrm{Da} \rightarrow \infty)$ are compared to those obtained by Job and Gunakala (2016) as shown in Table 2, and found that the observed results agree well with a relative error on the order of $0.56 \%$, and thereby validating the formulation and implementation of the proposed numerical scheme.

Table 2: Validation of code by comparing the average Nusselt number with Job and Gunakala (2016)

\begin{tabular}{lcc}
\hline Present code & Job and Gunakala (2016) & Relative error (\%) \\
\hline 4.6832 & 4.7100 & 0.56 \\
\hline
\end{tabular}

Thus, the numerical scheme has been validated by comparing the average Nusselt number with the results in literature. However, it may be noted that the computation of the average Nusselt number depends on the combined effect of Hartmann number, Darcy number, solid volume fraction and other processing parameters as well. Therefore, a sensitivity analysis was performed to see the influence of these parameters such as Hartmann number (Ha), Darcy number (Da), solid volume fraction on the average Nusselt number.

\section{Response surface methodology (RSM)}

The Response Surface Methodology (RSM) is one of the effective tools to describe several variables simultaneously with the least possible resources,

Table 3: Input variables and the levels of their values

\begin{tabular}{lcccc}
\hline Variable & Symbol & -1 & 0 & +1 \\
\hline Hartmann number & Ha & 25 & 50 & 100 \\
Darcy number & Da & $10^{-5}$ & $10^{-4}$ & $10^{-3}$ \\
Nanoparticles volume fraction & $\phi$ & 0.02 & 0.04 & 0.06 \\
\hline
\end{tabular}

Table 4: The levels of the parameters' values and the results of experiments for $N u_{a v}$

\begin{tabular}{|c|c|c|c|c|}
\hline \multirow[b]{2}{*}{ Run order } & \multicolumn{3}{|c|}{ Code values } & \multirow[b]{2}{*}{$N u_{a v}$} \\
\hline & $\mathrm{Ha}$ & $\mathrm{Da}$ & $\phi$ & \\
\hline 1 & -1 & -1 & -1 & 5.0532 \\
\hline 2 & 0 & 0 & 0 & 4.2894 \\
\hline 3 & 0 & 0 & 0 & 4.2894 \\
\hline 4 & -1 & -1 & 1 & 4.8774 \\
\hline 5 & 0 & 0 & 0 & 4.2894 \\
\hline 6 & -1 & 1 & -1 & 4.3753 \\
\hline 7 & 0 & 0 & 0 & 4.2894 \\
\hline 8 & 1 & 1 & -1 & 4.7011 \\
\hline 9 & 1 & -1 & -1 & 5.0593 \\
\hline 10 & 1 & -1 & 1 & 4.9771 \\
\hline 11 & 1 & 1 & 1 & 3.7894 \\
\hline 12 & -1 & 1 & 1 & 3.6092 \\
\hline 13 & 0 & 0 & 0 & 4.2894 \\
\hline 14 & -1 & 0 & 0 & 4.2316 \\
\hline 15 & 1 & 0 & 0 & 4.4693 \\
\hline 16 & 0 & 0 & -1 & 4.7030 \\
\hline 17 & 0 & 1 & 0 & 3.9650 \\
\hline 18 & 0 & 0 & 1 & 3.8884 \\
\hline 19 & 0 & 0 & 0 & 4.2894 \\
\hline 20 & 0 & -1 & 0 & 4.9680 \\
\hline
\end{tabular}


quantitative data and suitable test design, while the response variable is affected by many factors and variables.

The RSM presents some relationships between the several input variables and one or more response variables. For the sensitivity analysis, the Hartmann number (A), Darcy number (B) and solid volume fraction (C) were considered as input variables and average Nusselt number as a response variable. These variables are levelled with low $(-1)$, central $(0)$ and high $(+1)$. The range of the data is given in Table 3 (Box \& Wilson, 1951). We consider the second-degree model with interaction effects and apply the sequence of designed experiments for obtaining the optimal responses. This model can be summarised in the following mathematical relationship (Montgomery, 1996):

$$
\begin{aligned}
N u_{a v}= & \alpha_{0}+\alpha_{1} A+\alpha_{2} B+\alpha_{3} C+\alpha_{11} A^{2}+\alpha_{22} B^{2}+\alpha_{33} C^{2} \\
& +\alpha_{12} A B+\alpha_{23} B C+\alpha_{13} A C
\end{aligned}
$$

Equation (26) includes three linear terms (A, B and C), three squared terms $\left(\mathrm{A}^{2}, \mathrm{~B}^{2}\right.$, and $\left.\mathrm{C}^{2}\right)$, three two-factor interaction terms $(\mathrm{AB}, \mathrm{AC}$, and $\mathrm{BC})$ and one intercept term $\left(\alpha_{0}\right)$. Then, the dimensionless average Nusselt number, $N u_{a v}$ given in Table 6 was computed. The Central Composite Face centered design scheme (CCF) with $2^{a}+2 a+b$ experiments was employed to specify the response results. In the present analysis, twenty runs were considered (Table 4 ) with the number of factors, $a=3$ and the central points $b=6$.

Analysis of variance (ANOVA) was used for benchmarking the model accuracy in the Response Surface Methodology (RSM). ANOVA was conducted using MINITAB, and the ANOVA parameter values for adjusted mean squares, degrees of freedom (DOF), sum of squares, F value, $\mathrm{p}$ value, $R^{2}$, and adj $-R^{2}$ are tabulated in Table 5. The $\mathrm{F}$ value in this analysis is a measure of variance of data about the mean. The $5 \%$ level of significance for the statistical experiment is considered. The model is validated if the p-value calculated is less than 0.05 . From Table 5, all the $\mathrm{p}$ values obtained are significantly less than 0.05 . Therefore, statistically, this model is validated to be suitable for calculating the average Nusselt number. The values of $R^{2}$ and adj $-R^{2}$ indicate that the regression equation is fitted correctly.

The coefficients of linear, square and interaction variables are computed based on the results of CCF and the regression model reads:

$$
\begin{aligned}
N u_{a v}= & 4.2870+0.0809 A-0.4536 B-0.2710 C+0.0670 A^{2} \\
& +0.1830 B^{2}+0.0122 C^{2}+0.0449 A B-0.0014 A C \\
& -0.1724 B C
\end{aligned}
$$

The following points are noted from these results:

- Average Nusselt number is more sensitive to linear terms of Darcy number (B) and solid volume fraction (C).

- The small perturbation in the square of Darcy number $\left(\mathrm{B}^{2}\right)$ and the interaction of Darcy number and volume fraction (BC) causes significant variations in $N u_{a v}$. The derivative of the output variable to the input parameters are taken to analyse the behaviour of the

\begin{tabular}{|c|c|c|c|c|c|c|}
\hline Source & DOF & $\begin{array}{c}\text { Adjusted } \\
\text { sum of squares } \\
\text { (SS) }\end{array}$ & $\begin{array}{c}\text { Adjusted } \\
\text { mean squares } \\
\text { (MS) }\end{array}$ & $F$ value & $\mathrm{p}$ value & \\
\hline Model & 9 & 3.39342 & 0.37705 & 61.96 & 0.000 & Significant \\
\hline Linear & 3 & 2.85692 & 0.95231 & 156.49 & 0.000 & Significant \\
\hline Square & 3 & 0.28259 & 0.09420 & 15.48 & 0.000 & Significant \\
\hline 2-Way interaction & 3 & 0.25391 & 0.08464 & 13.91 & 0.001 & Significant \\
\hline Error & 10 & 0.06085 & 0.00609 & & & \\
\hline Lack-of-fit & 5 & 0.06085 & 0.01217 & * & * & \\
\hline Pure error & 5 & 0.00000 & 0.00000 & & & \\
\hline Total & 19 & 3.45428 & & & & \\
\hline
\end{tabular}
sensitivity of the mean Nusselt number to each parameter and written as

$$
\frac{\partial N u_{a v}}{\partial A}=0.0809+0.1340 A+0.0449 B-0.0014 C
$$

Table 5: The variance analysis of $N u_{a v}$

$R^{2}=98.24 \% ;$ adj $-R^{2}=96.65 \%$ 


$$
\begin{aligned}
& \frac{\partial N u_{a v}}{\partial B}=-0.4536+0.366 B+0.0449 A-0.1724 C \\
& \frac{\partial N u_{a v}}{\partial C}=-0.2710+0.0244 C-0.0014 A-0.1724 B
\end{aligned}
$$

The positive value of sensitivity indicates increasing of objective function due to the increase of input parameters. Moreover, the negative value of sensitivity represents a decrease in objective function caused by an increase in the input parameters. The sensitivity of the objective function with respect to $H a, D a$ and $\phi$ are tabulated (Table 6).

Table 6: The sensitivity analysis of $\bar{N} u$

\begin{tabular}{cccccc}
\hline \multicolumn{6}{c}{ Sensitivity } \\
$\mathrm{A}$ & $\mathrm{B}$ & $\mathrm{C}$ & $\frac{\partial N u_{a v}}{\partial A}$ & $\frac{\partial N u_{a v}}{\partial B}$ & $\frac{\partial N u_{a v}}{\partial C}$ \\
\hline 0 & 0 & -1 & 0.0823 & -0.2812 & -0.2954 \\
0 & 0 & 0 & 0.0809 & -0.4536 & -0.2710 \\
0 & 0 & 1 & 0.0795 & -0.6260 & -0.2466 \\
1 & 0 & -1 & 0.2163 & -0.2363 & -0.2968 \\
1 & 0 & 0 & 0.2149 & -0.4087 & -0.2724 \\
1 & 0 & 1 & 0.2135 & -0.5811 & -0.2480 \\
\hline
\end{tabular}

From the tabulated results (Table 6), the following conclusions may be drawn:

- The sensitivity of $N u_{a v}$ to Hartmann number is positive, but it decreases with the increase of the fraction of nanoparticles.

- The influence of the solid volume fraction is small at a high Hartman number flow.

- The sensitivity of $N u_{a v}$ to Darcy number is negative, but it increases with the increase of the $\phi$

- The sensitivity of $N u_{a v}$ to volume fraction is negative, but its magnitude decreases with the increase of the $\phi$

It is concluded that $N u_{a v}$ is more sensitive to Darcy number and the sensitivity of the mean Nusselt number $N u_{a v}$ to Darcy number will be more with the addition of nanoparticles into the cavity.

\section{RESULTS AND DISCUSSION}

The fluid is at rest at the initial stage. As time advances, the temperature difference between the wavy wall and the side walls induces the flow. The clockwise and anticlockwise flow circulations take place separated by the symmetry of the wavy wall.
From Figure 2, it can be observed that streamline plots and isotherms are symmetrical about the center vertical line of the enclosure. It is observed from the streamline plots that the flow pattern has secondary circulation cells symmetrically beside the bottom wall at small times and primary cells cover the remaining enclosure. With the advancement of time, the secondary small circulation cells disappear and finally flow circulation takes place through two primary cells, which are on both sides of symmetry. It is also clear that the maximum circulation intensity $|\psi|$ increases as time is increasing and finally reaches steady state. It is further seen that the flow circulation profiles are identical to the one reported in Job and Gunakala (2016). However, the flow circulation strength is small in the present study due to the porous effect.

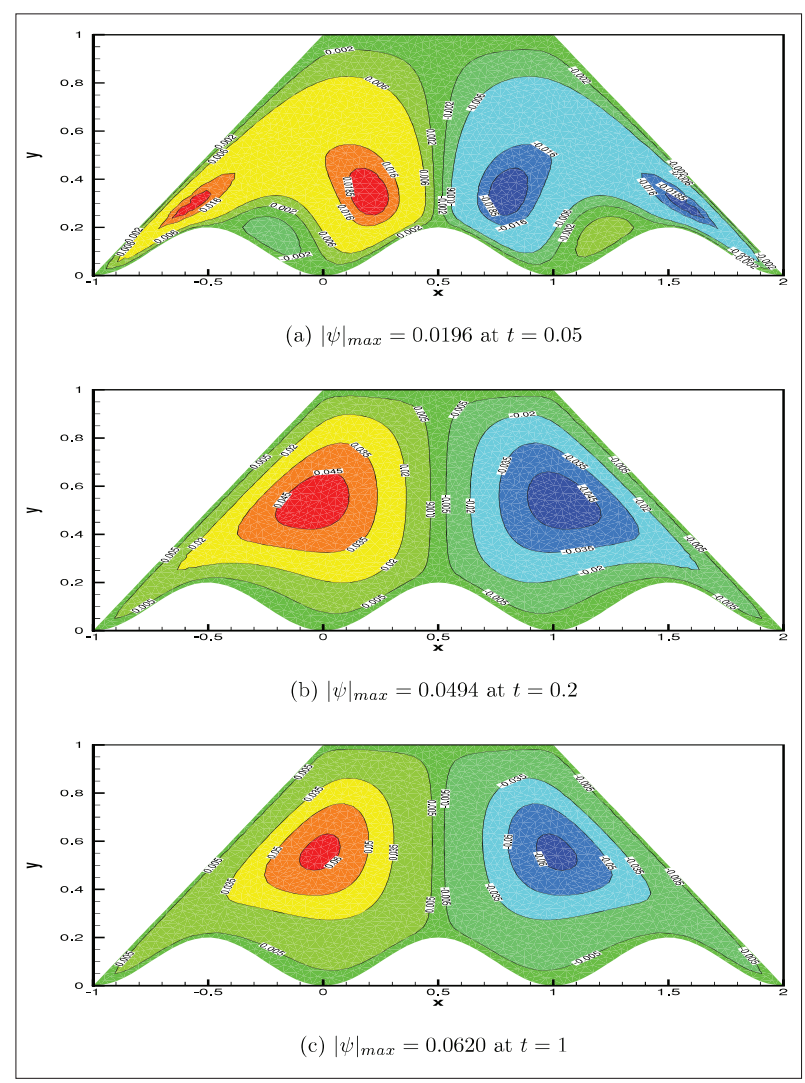

Figure 2: Streamlines at different times $t$ for $D a=10^{-4} ; \phi=0.02$; $\mathrm{Ha}=100$

Figure 3 represents the isotherm plots, which show that temperature distribution moving from wavy bottom wall to inside the enclosure as time increases. Heat transfer is taking place from the heated wavy bottom wall to the cold sided walls and hence towards the top insulated wall. It can be seen that as time increases the temperature 


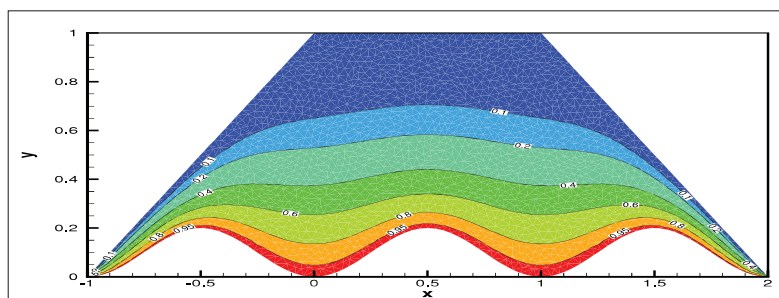

(a) $t=0.05$

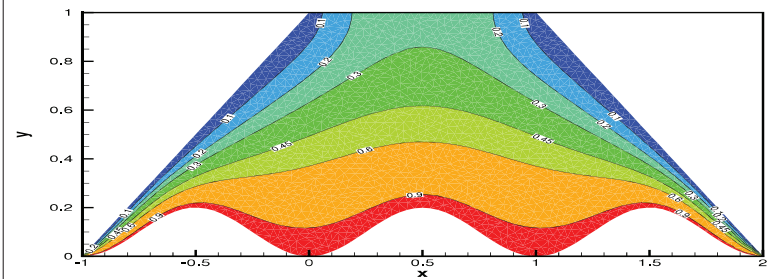

(b) $t=0.2$

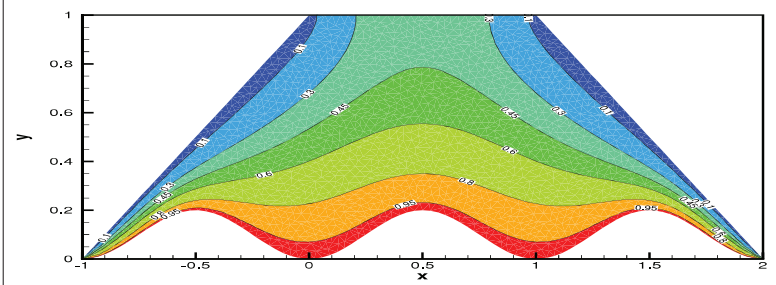

(c) $t=1$

Figure 3: Isotherm plots at different times $t$ for $D a=10^{-4} ; \phi=0.02$; $H a=100$

gradient decreases near the bottom wall. Physically it means that temperature distribution reduces and reaches to steady state. It can be further observed that the trends in temperature distributions are similar to the one reported in Job and Gunakala (2016). Since the flow strength inside the enclosure reduces as the fluid experience resistance due to the presence of porous domain, the movement of temperature from the wavy bottom wall to the insulated wall is relatively less in comparison to the flow in the non-porous medium for the natural convection flow.

The nanofluid flow under the porous medium has its significance in its circulation and heat transfer. Figure 4 shows the difference in maximum circulation intensity, which is caused due to the change in the number of nanoparticles dispersed in the base fluid which is expressed as a ratio, the solid volume fraction $\phi$.

One can observe that $\mathrm{Al}_{2} \mathrm{O}_{3}$ nanoparticles have less electrical conductivity than the electrical conductivity of a base fluid, which means Lorentz force reduces with an increase of the concentration of nanoparticles. Although

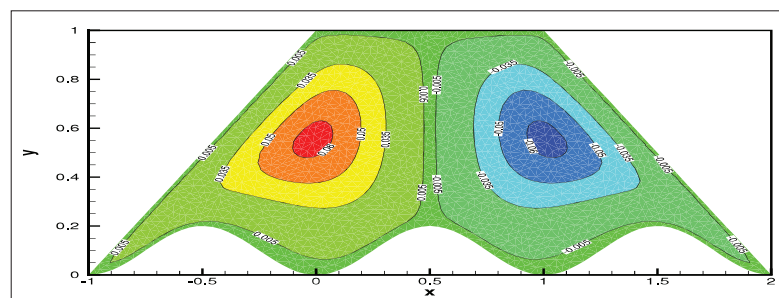

(a) $|\psi|_{\max }=0.0620$ for $\phi=0.02$

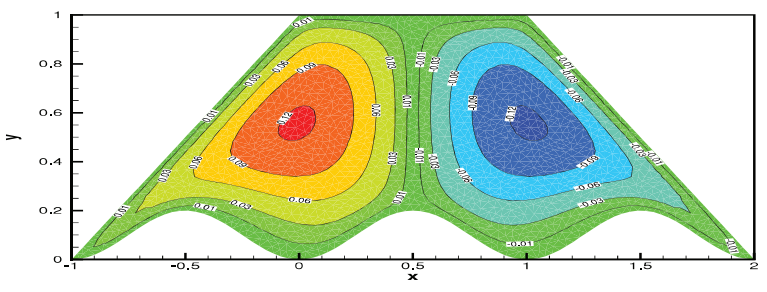

(b) $|\psi|_{\max }=0.1236$ for $\phi=0.04$

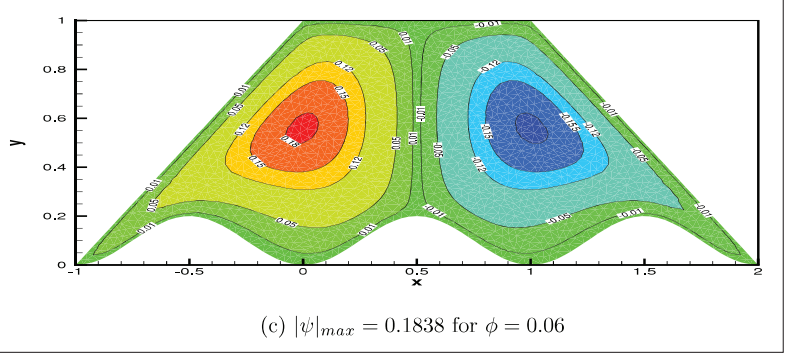

Figure 4: Streamlines for different values of $\phi$ for $D a=10^{-4} ; t=1$; $H a=100$

the Lorentz force applied to nanoparticles is weak, the thermal expansion coefficient is very high in these nanoparticles, which resulted in increased circulation strength of the fluid. It is to be noted that the flow circulation intensity is small in comparison to the flow in a non-porous domain (Job \& Gunakala, 2016).

It can be observed that the nanoparticles have more thermal conductivity than base fluid. Therefore, an increase in the concentration leads to an increase in viscous dissipation and Joule heating, and thereby temperature distribution increases. It can also be seen from Figure 5 that heat transfer takes place from the wavy bottom wall to the top wall.

Permeability (K) in fluid mechanics gives the estimate of the capacity of a porous material to let fluids to go through it. The higher the Darcy number, the measure of the ability of a porous material to allow fluid to flow is high, and hence the momentum of fluid increases. This means that velocity of the fluid increases along with maximum circulation intensity as shown in Figure 6. 


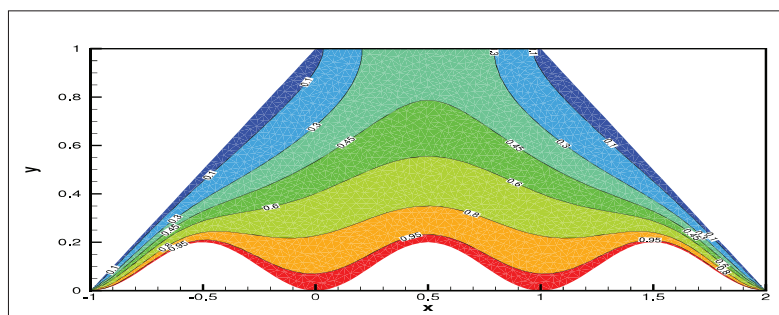

(a) $\phi=0.02$

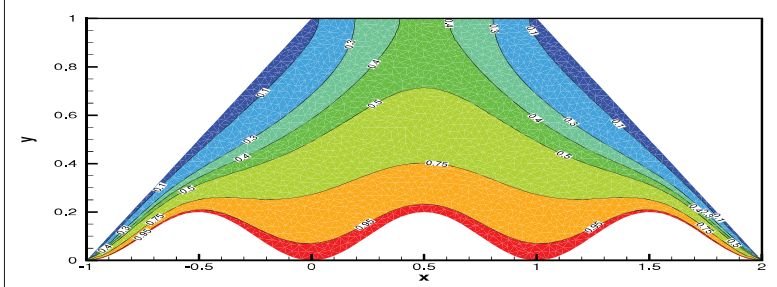

(b) $\phi=0.04$

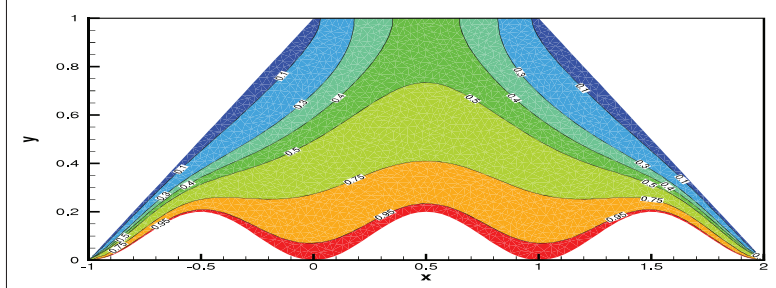

(c) $\phi=0.06$

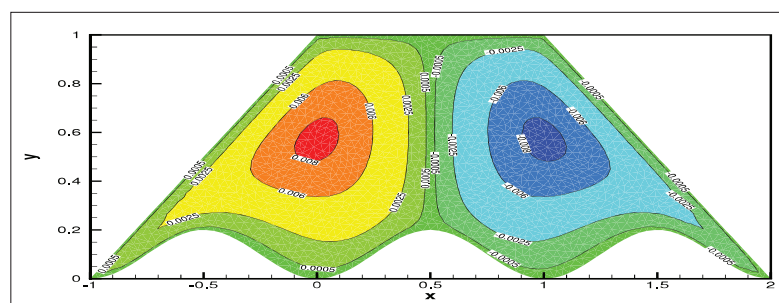

(a) $|\psi|_{\max }=0.0083$ for $D a=10^{-5}$

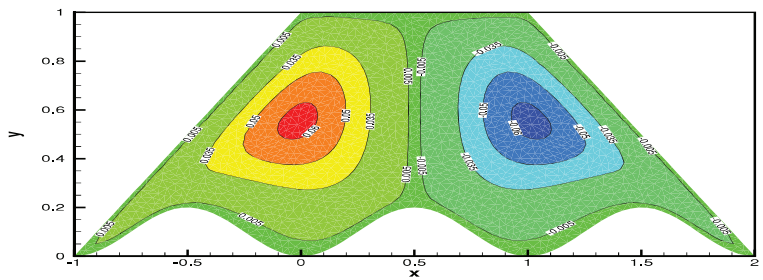

(b) $|\psi|_{\max }=0.0620$ for $D a=10^{-4}$

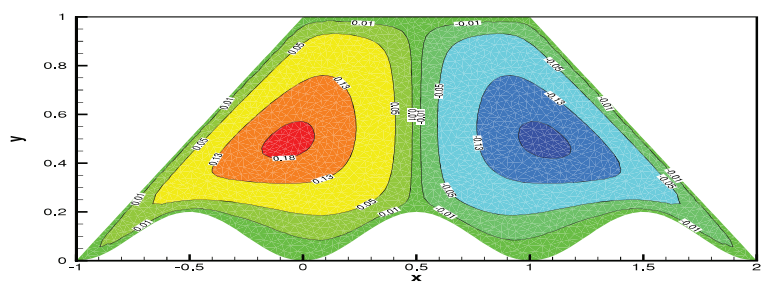

(c) $|\psi|_{\max }=0.1887$ for $D a=10^{-3}$

Figure 5: Isotherm plots for different values of $\phi$ for $D a=10^{-4} ; t=1$; $H a=100$

Figure 6: Streamlines for different values of $D a$ for $\phi=0.02 ; t=1$; $H a=100$

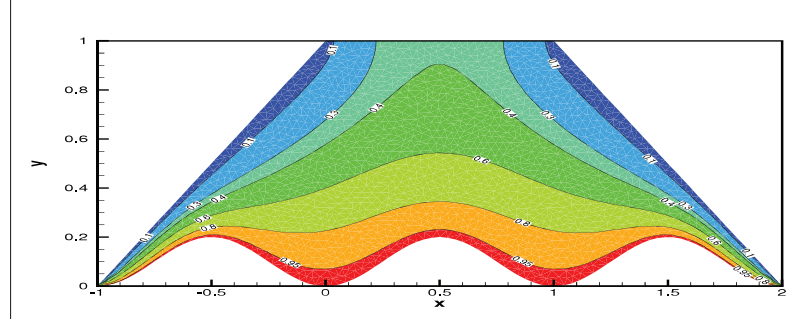

(a) $D a=10^{-5}$

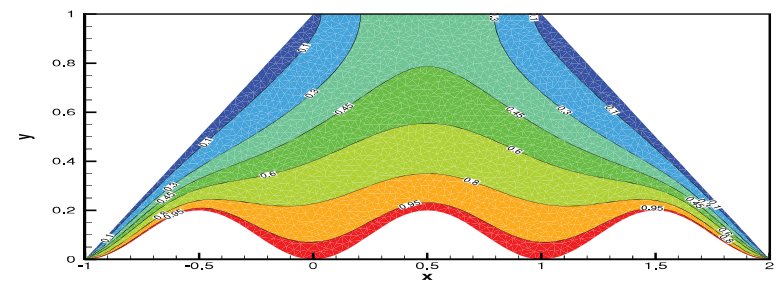

(b) $D a=10^{-4}$

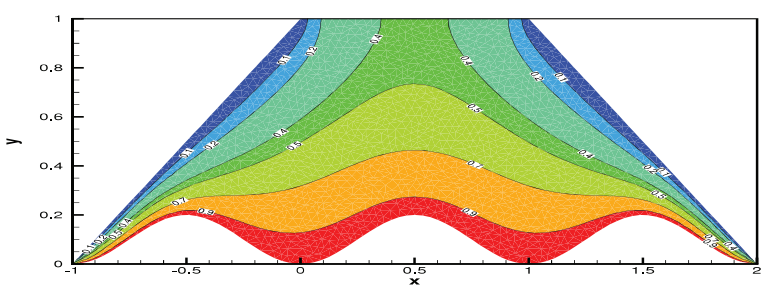

(c) $D a=10^{-3}$

Figure 7: Isotherm plots for different values of $D a$ for $\phi=0.02 ; t=1 ; H a=100$ 
With respect to isotherm (Figure 7), when the Darcy number is higher $\left(D a=10^{-3}\right)$, the heat convection dominates, and the heat propagates faster towards the insulated wall from the bottom heated wavy wall. For example, when $D a$ is smaller $\left(D a=10^{-5}\right)$, there is more drag (viscous) in the flow, and hence the heat transfer is dominated by conduction effect.

The average heat transfer rate through the wavy bottom wall is presented in Table 7. It is found that the heat transfer rate increases with a decrease in Darcy number. A decrease in Darcy number ceases the flow circulation ability, resulting in the heat to transfer due to viscous friction. As $D a$ decreases, the heat transfer dominates by heat conduction.

Table 7: Average Nusselt number $N u_{a v}$ for $H a=100 ; \operatorname{Pr}=6.2$; $E c=10^{-5} ; a=0.1 \phi=0.02$ and $R a=100$ at $t=1$

\begin{tabular}{ll}
\hline $\mathrm{Da}$ & $N u_{a v}$ \\
\hline $10^{-5}$ & 5.0593 \\
$10^{-4}$ & 4.8543 \\
$10^{-3}$ & 4.7011 \\
\hline
\end{tabular}

One can observe that if the permeability goes to infinite, then the average Nusselt number reaches the value that is validated in Table 2 which means porous medium has no significance in fluid flow.

\section{CONCLUSION}

In the present work, the natural convection fluid flow and heat transfer in a porous trapezoidal enclosure that has a wavy bottom wall filled with $\mathrm{Al}_{2} \mathrm{O}_{3}$-water nanofluid in the presence of magnetic field was discussed. The model equations were solved numerically using finite element method. The implementation is compared with earlier published work as a special case for non-Darcy flow $(D a \rightarrow \infty)$. The effects of time, Darcy number, solid volume fraction on streamlines and isotherms were discussed. The average Nusselt number was investigated for different values of Darcy number and observed that the heat transfer rate increases due to the decrease in permeability. In conclusion, the flow circulation and fluid temperature inside the enclosure increased with time. Heat transfer rate near the wavy bottom wall decreased with time and then reached the steady state. The flow circulation and temperature of the fluid inside the trapezoidal enclosure increased with the increase in the volume fraction of nanoparticles. However, the temperature and stream function values of the nanofluid under the porous medium are small compared to the nanofluid, which was not under porous medium. Moreover, the sensitivity study revealed that the average Nusselt number is more sensitive to the Darcy number.

\section{REFERENCES}

Alhashash A., Saleh H. \& Hashim I. (2014). Natural convection in a porous enclosure filled with nanofluids. AIP Conference Proceedings 1614: 823-826.

DOI: https://doi.org/10.1063/1.4895308

Al-Hadhrami A.K., Elliott L. \& Ingham D.B. (2003). A new model for viscous dissipation in porous media across a range of permeability values. Transport in Porous Media 53: $117-122$.

DOI: https://doi.org/10.1023/A:1023557332542

Box G.E.P. \& Wilson K.B. (1951). On the experimental attainment of optimum conditions. Journal of the Royal Statistical Society. Series B (Methodological) 13: 1-45. DOI: https://doi.org/10.1111/j.2517-6161.1951.tb00067.x

Cho C.C., Yau H.T. \& Kuang Chen C. (2012). Numerical investigation into natural convection heat transfer enhancement of copper-water nanofluid in a wavy wall enclosure. Thermal Science 16(5): 1309-1316. DOI: https://doi.org/10.2298/TSCI1205309C

Choi S.U.S. \& Eastman J.A. (1995). Enhancing thermal conductivity of fluids with nanoparticles. Developments and Applications of Non-Newtonian Flows 66: 99-105.

Daungthongsuk W. \& Wongwises S. (2009). A critical review of convective heat transfer in nanofluids. Renewable and Sustainable Energy Reviews 11: 797-817.

DOI: https://doi.org/10.1016/j.rser.2005.06.005

Donea J. \& Huerta A. (2003). Finite Element Methods for Flow Problems. John Wiley and Sons, Ltd., New Jersey, USA. DOI: https://doi.org/10.1002/0470013826

Hwang K.S., Lee J.H. \& Jang S.P. (2007). Buoyancy-driven heat transfer of water-based $\mathrm{Al}_{2} \mathrm{O}_{3}$ nanofluids in a rectangular cavity. International Journal of Heat and Mass Transfer 50(19): 4003-4010.

Job V.M. \& Gunakala S.R. (2016). Unsteady MHD free convection nanofluid flows within a wavy trapezoidal enclosure with viscous and joule dissipation effects. Numerical Heat Transfer, Part A: Applications 69(4): 421443.

DOI: https://doi.org/10.1080/10407782.2015.1080946

Leonard M., Mozumder A.K., Mahmud S. \& Das P.K. (2017). Natural convection of $\mathrm{Al}_{2} \mathrm{O}_{3}$-water nanofluid in a wavy enclosure. AIP Conference Proceedings 1851: 020003. DOI: https://doi.org/10.1063/1.4984632

Mahmoudi A.H., Pop I., Shadi M. \& Talebi F. (2013). MHD natural convection and entropy generation in a trapezoidal enclosure using $\mathrm{Cu}$-water nanofluid. Computers and Fluids 72: 46-62.

Minkowycz W.J., Sparrow E.M. \& Abraham J.P. (2013). Nanoparticle Heat Transfer and Fluid Flow, $4^{\text {th }}$ edition. CRC Press, New York, USA. 
Montgomery D.C. (1996). Design and Analysis of Experiments. John Wiley \& Sons, Ltd., New Jersey, USA.

Qu W., Mala Gh.M. \& Li D. (2000). heat transfer for water flow in trapezoidal silicon micro channels. International Journal of Heat and Mass Transfer 43: 3925-3936.

Rashidi S., Bovand M. \& Esfahani J.A. (2015a). Heat transfer enhancement and pressure drop penalty in porous solar heat exchangers: A sensitivity analysis. Energy Conversion and Management 103: 726-738. DOI: https://doi.org/10.1016/j.enconman.2015.07.019

Rashidi S., Bovand M. \& Esfahani J.A. (2015b). Structural optimization of nanofluid flow around an equilateral triangular obstacle. Energy 88: 385-398. DOI: https://doi.org/10.1016/j.energy.2015.05.056

Rashidi S., Bovand M., Esfahani J.A. \& Ahmadi G. (2016). Discrete particle model for convective $\mathrm{Al}_{2} \mathrm{O}_{3}$-water nanofluid around a triangular obstacle. Applied Thermal Engineering 100: 39-54.

DOI:https://doi.org/10.1016/j.applthermaleng.2016.01.076

Reddy J.N. (2004). An Introduction to Nonlinear Finite Element Analysis. Oxford University Press, Oxford, UK.

Roslan R., Saleh H. \& Hashim I. (2011). Buoyancy-driven heat transfer in nanofluid- filled trapezoidal enclosure with variable thermal conductivity and viscosity. Numerical Heat Transfer, Part A: Applications 60: 867-882. DOI: https://doi.org/10.1080/10407782.2011.616778

Sadoughi K., Hosseini M., Shakeri F. \& Azimi M. (1973). Analytical simulation of MHD nanofluid flow over the horizontal plate. Frontiers in Aerospace Engineering 2: 242-246.

Salari M., Malekshah E.H. \& Malekshah M.H. (2017). Natural convection in a rectangular enclosure filled by two immiscible fluids of air and $\mathrm{Al}_{2} \mathrm{O}_{3}$ - water nanofluid heated partially from side walls. Alexandria Engineering Journal 57(3): 1401-1412.

DOI: https://doi.org/10.1016/j.aej.2017.07.004

Singh S. \& Bhargava R. (2014). Numerical study of natural convection with a wavy enclosure using meshfree approach: effect of corner heating. Scientific World Journal 2014: Article ID 842401.

DOI: https://doi.org/10.1155/2014/842401

Suh J., Greif R. \& Grigoropoulos C.P. (2001). Friction in micro-channel flows of a liquid and vapor in trapezoidal and sinusoidal grooves. International Journal of Heat and Mass Transfer 44: 3103-3109.

Taylor C. \& Hood P. (1973). A numerical solution of the Navier-Stokes equations using the finite element technique. Computers and Fluids 1 (1): 73-100.

Ternik P. \& Rudilf R. (2014). Conduction and convection heat transfer characteristics of water-based Au nanofluids in a square cavity with differentially heated side walls subjected to constant temperatures. Thermal Science 18: 189-200. DOI: https://doi.org/10.2298/TSCI130604082T

Ternik P., Rudolf R. \& Zunic Z. (2012). Numerical study of heat transfer enhancement of homogeneous water-Au nanofluid under natural convection. Materials Technology 46: $257-261$. 